Rev. salud pública. 9 (4):609-617, 2007

Ensayo/Essay

\title{
La Metasíntesis: una Metodología de Investigación
}

\author{
Meta-synthesis: a research methodology
}

Gloria M. Carrillo-González, Olga J. Gómez-Ramírez y Elizabeth Vargas-Rosero

Departamento de Cuidado y Práctica de Enfermería, Facultad de Enfermería, Universidad

Nacional de Colombia. gmcarrillog@unal.edu.co, ojgomezr@unal.edu.co, evargasr@unal.edu.co

Recibido 29 Enero 2007/Enviado para Modificación 30 Septiembre 2007/Aceptado 15 Octubre 2007

\section{RESUMEN}

Este artículo es el resultado de la revisión temática de fuentes primarias acerca de la metasíntesis, considerada como una metodología investigativa novedosa para las ciencias de la salud, que permite valorar la evidencia científica de la investigación cualitativa y fortalecer el cuerpo de conocimientos de una disciplina al construir, describir y/o explicar fenómenos. Se presentan las definiciones de metasíntesis propuestas por varios autores que coinciden en afirmar que el propósito de ésta es aumentar la interpretación de los resultados de estudios cualitativos generando impacto en la asistencia, la investigación y dando respuesta a los problemas de la práctica. Finalmente, se plantean algunos criterios a tener en cuenta al aplicar la metodología.

Palabras Clave: Enfermería, investigación cualitativa, literatura de revisión (fuente: DeCS, BIREME).

\section{ABSTRACT}

This article resulted from a bibliographical review concerning meta-synthesis, considered to be a novel methodology in health science research. Meta-synthesis' importance lies in evaluating high complex scientific evidence from qualitative research and strengthening a discipline's body of knowledge when constructing, describing or explaining related theories. Several authors' definitions of metasynthesis are presented. They coincide in stating that its purpose is to enhance interpreting an outcome by integrating the findings from qualitative research and thus have an impact on support, research and proposals in resolving problems regarding nursing practice. Some criteria to be born in mind when applying this methodology are also mentioned.

Key Words: Nursing, qualitative research, review literature (source: MeSH, NLM). 
REVISTADE SALUD PÚBLICA· Volumen 9 (4), Diciembre 2007

$\mathrm{P}$ ara esta revisión temática se realizo una búsqueda en las bases de datos Ebsco, Lilacs, Ovid, Proquest, Hinari, Medline, Mediclatina y Pubmed. Se incluyeron como palabras de búsqueda en todas las combinaciones posibles los términos: meta-synthesis, qualitative meta-synthesis, qualitative meta-analysis, qualitative research evidence y su equivalente en español. Se incluyeron artículos publicados desde 1984 hasta 2006. Este proceso se complemento con una revisión directa de cada una de las referencias que citaban las publicaciones encontradas y con la identificación de recursos adicionales como capítulos de libros en búsqueda de información relevante. Todos los artículos incluidos fueron revisados por los autores.

La metasíntesis constituye una metodología novedosa de gran utilidad que brinda a través de sus métodos y tipos; elementos para construir, describir o explicar teorías en relación a los fenómenos de interés para las disciplinas. Es un tipo de evidencia científica de gran complejidad que implica rigor en la selección del conocimiento tal como lo indica Gálvez (1): "...el nivel más alto de complejidad de la investigación secundaria cualitativa es la metasíntesis y se define como la integración y síntesis de hallazgos, realizada a partir de varios metaestudios cualitativos".

¿Qué es metasíntesis?

En esta revisión se indentificaron varias conceptualizaciones de metasíntesis que están relacionadas con una multiplicidad de términos usados para describir los hallazgos cualitativos. Schreiber $(2,3)$ indica que el término meta-análisis cualitativo o metasíntesis fue utilizado por primera vez en 1985 por Stern y Harris para referirse a la síntesis de un grupo de hallazgos de investigaciones cualitativas dentro de un producto interpretativo y explicativo. Noblit $(4,5)$ ha sintetizado hallazgos etnográficos que ha llamado metaetnografia. Estarboocks utilizó el término "agregando hallazgos" y consideró que esto significa incrementar el nivel de abstracción y de esta manera desarrollar teoría de mediano rango.

Según Gálvez (7-10) la metasíntesis es un completo estudio que involucra una revisión e interpretación rigurosa de los hallazgos de un número de investigaciones cualitativas, usando métodos cualitativos. Tiene como meta producir una interpretación nueva e integrativa de los hallazgos, que aporte mucho más que los resultados individuales de cada investigación.

Gu Jifa (11) define la metasíntesis como un sistema de conocimiento abierto que pretende la integración de datos, teorías, métodos, hallazgos y cualquier 
otro tipo de conocimiento para dar respuesta a problemas complejos. Finfgeld (12) plantea que esta metodología permite la clarificación de conceptos, patrones y resultados para el refinamiento de estados del conocimiento existentes y el surgimiento de nuevos modelos y teorías. Adicionalmente, estos tienen el potencial de mejorar los resultados clínicos, realizar investigaciones y formular políticas para el cuidado de la salud.

Tipos de metasíntesis: Finfgeld (12-14) plantea tres tipos de metasíntesis que son complementarias: la construcción de teoría, explicación de teoría y descripción de teoría.

1. Construcción de la teoría: Se utiliza para mejorar el nivel de la teoría, más allá de lo que cual es posible con una sola investigación. En esta se pueden diferenciar dos tipos de metasíntesis.

1.1. Teoría formal fundamentada. En la que los hallazgos de investigaciones individuales son analizados a través de métodos estándar de teoría fundamentada y después se sintetizan en estudios para desarrollar un modelo más comprehensivo (extenso) del fenómeno. Kearney (15-18).

1.2 Meta- estudio. Se compone de tres tipos de análisis: metadatos (análisis e interpretación de los resultados de investigaciones cualitativas diversas), metamétodo (estudio del rigor y validez epistemológica de los métodos usados para conducir los estudios cualitativos) y metateoría (un análisis de las perspectivas filosóficas, cognoscitivas y teóricas).

Schreiber (19) plantea que los supuestos esenciales del problema son los datos y la utilidad potencial del producto final son la creación de teoría, generación de preguntas de investigación e identificación de vacíos en el conocimiento. La metodología es inductiva y los datos son los resultados de estudios de fenómenos o conceptos relacionados que se analizan por comparación constante, lo cual permite asegurar el rigor científico.

2. Explicación de la teoría: Usando la explicación de la teoría los conceptos abstractos son "sacados a la luz", dando como resultado la reconceptualización del fenómeno original.

El propósito en la explicación de teoría es desarrollar teoría de mediano rango de un grupo de estudios de un sólo concepto relacionado, los supuestos esenciales del problema conducen a hallazgos de conceptos que pueden ser reconstruidos. 
REVISTADE SALUD PÚBLICA · Volumen 9 (4), Diciembre 2007

Los límites del estudio son predefinidos y la metodología es deductiva. Los datos se analizan por reconstrucción y comparación constante. El procedimiento que se utiliza para el muestreo puede iniciarse con conceptos abstractos y continuar relacionando conceptos menos abstractos (19).

3. Descripción de teoría: Implica la síntesis de resultados y hallazgos cualitativos en un análisis más extensivo del fenómeno. En contraste con la teoría explicativa, la cual se centra en el análisis de un solo concepto, la metasíntesis descriptiva mira más ampliamente los fenómenos, en el que los resultados no se alteran, pues no se deconstruyen para ser analizados.

Finfgeld (12) concluye que los tres modelos mencionados no reflejan necesariamente todas las posibilidades de la metasíntesis, son simplemente un mecanismo para pensar como los estudios han sido desarrollados en el pasado y las formas en que pueden ser desarrollados en el futuro. Tabla 1: Terminología en Metasíntesis.

Tabla 1. Terminología en Metasíntesis

\begin{tabular}{|c|c|}
\hline Termino & Significado \\
\hline $\begin{array}{l}\text { Teoria formal } \\
\text { fundamentada }\end{array}$ & $\begin{array}{l}\text { Investigación inductiva que usa los hallazgos } \\
\text { significativos de los estudios de teoria fundamentada para } \\
\text { sintetizar una nueva teoria formal fundamentada (15). }\end{array}$ \\
\hline Metanálisis & $\begin{array}{l}\text { Termino frecuentemente u sado para describir la } \\
\text { combinación estadistica y análisis de resultados de } \\
\text { múltiples estudios cuantitativos (20). }\end{array}$ \\
\hline Metaestudio & $\begin{array}{l}\text { Investigación inductiva que se aproxima al análisis e } \\
\text { interpretación de teoria, métodos y hallazgos de } \\
\text { investigación a través de estudios cualitativos y de } \\
\text { sintesis de estos trabajos para formular nuevas } \\
\text { interpretaciones }\end{array}$ \\
\hline Análisis de Metadatos & $\begin{array}{l}\text { Análisis e interpretación de hallazgos a través de } \\
\text { múltiples reportes de investigación cualitativa. }\end{array}$ \\
\hline Metamétodo & $\begin{array}{l}\text { Análisis e interpretación de aplicaciones metodológicos a } \\
\text { través de múltiples reportes de investigación cualitativa. }\end{array}$ \\
\hline Metateoria & $\begin{array}{l}\text { Análisis e interpretación de perspectivas teóricas, } \\
\text { filosóficas y cognitivas; fuentes y contextos a través de } \\
\text { múltiples estudios cualitativos (21). }\end{array}$ \\
\hline Metasintesis & $\begin{array}{l}\text { Término referido a las sintesis de hallazgos cualitativos } \\
\text { para la creación y nueva interpretación. Los tipos de } \\
\text { metasintesis incluyen: Construcción de teoria, } \\
\text { metaestudio, teoria formal fundamentada, teoria } \\
\text { explicativa y descripción de teoria. }\end{array}$ \\
\hline
\end{tabular}

Metodología utilizada en la metasíntesis

Delimitación del estudio: cuando se realiza una metasíntesis surgen inquietudes filosóficas que pueden afectar la opción metodológica tomada. Según Schreiber 
$(2,3)$ "El investigador debe determinar el propósito y los resultados esperados del análisis a partir de los tipos de metasíntesis ya descritos, debe decidir qué datos serán considerados, cómo se incluirá la literatura en la muestra, además de la determinación de los criterios de inclusión y exclusión". Se debe determinar cuales serán los datos sujetos al análisis, decidir qué será incluido y porqué, para determinar el alcance del estudio, como se indica en la Tabla 2 (2).

Tabla 2. Modelos propuestos de investigación en Metasíntesis (2)

\begin{tabular}{|c|c|c|c|}
\hline \multirow[b]{2}{*}{$\begin{array}{l}\text { Problemas } \\
\text { jpreguntas }\end{array}$} & \multicolumn{3}{|c|}{ MODELO } \\
\hline & Construcción de teoría & Explicación de teoria & Descripción de teoría \\
\hline $\begin{array}{l}\text { ¿Cuál es el propósito } \\
\text { de este meta- } \\
\text { análisis? }\end{array}$ & $\begin{array}{l}\text { Construir teoria de medio } \\
\text { rango o formal de un grupo } \\
\text { de estudios de conceptos } \\
\text { relacionados. }\end{array}$ & $\begin{array}{l}\text { Desarrollar teoría de } \\
\text { medio rango o teoria } \\
\text { formal de un grupo de } \\
\text { estudios de un solo } \\
\text { concepto relacionado. }\end{array}$ & $\begin{array}{l}\text { Examinar un grupo de } \\
\text { estudios que descubren } \\
\text { la interpretación } \\
\text { naturalista esencial o el } \\
\text { entendimiento de un } \\
\text { fenómeno. }\end{array}$ \\
\hline $\begin{array}{l}\text { ¿Cuáles son los } \\
\text { supuestos esenciales } \\
\text { del problema? }\end{array}$ & Todos son datos. & $\begin{array}{l}\text { Los hallazgos pueden } \\
\text { ser reconstruidos. }\end{array}$ & $\begin{array}{l}\text { El análisis final está en } \\
\text { el texto. }\end{array}$ \\
\hline $\begin{array}{l}\text { ¿Cuál es la utilidad } \\
\text { potencial del } \\
\text { producto final? }\end{array}$ & $\begin{array}{l}\text { Creación de teoria. } \\
\text { Generación de preguntas } \\
\text { de investigación. } \\
\text { Identificación de vacios en } \\
\text { el conocimiento. }\end{array}$ & $\begin{array}{l}\text { Desarrollo de teoría. } \\
\text { Generación de } \\
\text { preguntas de } \\
\text { investigación. } \\
\text { Identificación de vacios } \\
\text { en el conocimiento. }\end{array}$ & $\begin{array}{l}\text { Información para la } \\
\text { práctica. } \\
\text { Información para la } \\
\text { formulación de politicas. } \\
\text { Identificación de vacios } \\
\text { en el conocimiento. } \\
\text { Descubrir la teoría } \\
\text { esencial. }\end{array}$ \\
\hline $\begin{array}{l}\text { ¿Cuáles son los } \\
\text { limites del estudio? }\end{array}$ & $\begin{array}{l}\text { Los limites son emergentes } \\
\text { y siguen el desarrollo de los } \\
\text { resultados del estudio. }\end{array}$ & $\begin{array}{l}\text { Los limites son } \\
\text { predefinidos. }\end{array}$ & $\begin{array}{l}\text { Los limites pueden ser } \\
\text { predefinidos o } \\
\text { emergentes. }\end{array}$ \\
\hline $\begin{array}{l}\text { ¿Cuáles son los } \\
\text { supuestos principales } \\
\text { de la metodologia del } \\
\text { meta-análisis? }\end{array}$ & $\begin{array}{l}\text { Inductivo/emergente se } \\
\text { aproxima a la construcción } \\
\text { de teoria. }\end{array}$ & $\begin{array}{l}\text { Deductiva y se } \\
\text { aproxima a la } \\
\text { construcción de teoria. }\end{array}$ & $\begin{array}{l}\text { La teoria no } \\
\text { necesariamente } \\
\text { produce los resultados } \\
\text { deseados. Los } \\
\text { hallazgos se } \\
\text { fundamentan y se } \\
\text { cierran los datos. }\end{array}$ \\
\hline $\begin{array}{l}\text { ¿Qué constituye los } \\
\text { datos? }\end{array}$ & $\begin{array}{l}\text { Estudios de fenómenos o } \\
\text { conceptos relacionados. }\end{array}$ & $\begin{array}{l}\text { Estudios de igual } \\
\text { concepto. }\end{array}$ & $\begin{array}{l}\text { Puede usarse cualquier } \\
\text { estudio cualitativo. }\end{array}$ \\
\hline $\begin{array}{l}\text { ¿Cómo se analizarán } \\
\text { los datos? }\end{array}$ & Por comparación constante. & $\begin{array}{l}\text { Por reconstruoción, } \\
\text { reconstrucción y } \\
\text { comparación constante. }\end{array}$ & $\begin{array}{l}\text { Puede usarse } \\
\text { fenomenologia, } \\
\text { hermenéutica o } \\
\text { etnografia. }\end{array}$ \\
\hline $\begin{array}{l}\text { ¿Qué procadimiento } \\
\text { se usa para el } \\
\text { muestreo? ¿Como } \\
\text { saber qué incluir? }\end{array}$ & $\begin{array}{l}\text { Se puede empezar en } \\
\text { cualquier parte y continuar } \\
\text { a través de niveles más } \\
\text { altos de abstracción. } \\
\text { Estudios relacionados por } \\
\text { conceptos. }\end{array}$ & $\begin{array}{l}\text { Puede empezarse con } \\
\text { conceptos más } \\
\text { abstractos y continuar } \\
\text { relacionando conceptos } \\
\text { menos abstractos. } \\
\text { Estudios relacionados } \\
\text { por un solo concepto. }\end{array}$ & $\begin{array}{l}\text { Puede empezarse con } \\
\text { una categoría } \\
\text { diagndstica (personas } \\
\text { con cáncer de seno por } \\
\text { ejemplo). }\end{array}$ \\
\hline $\begin{array}{l}\text { ¿Cómo asegurar el } \\
\text { rigor cientifico del } \\
\text { meta-análisis? }\end{array}$ & $\begin{array}{l}\text { La comparacion constante } \\
\text { permitira a la variable } \\
\text { central emerger. }\end{array}$ & $\begin{array}{l}\text { La metodologia debe } \\
\text { tener una observación } \\
\text { cuidadosa. }\end{array}$ & $\begin{array}{l}\text { Los hallazgos deben ser } \\
\text { inmediatamente } \\
\text { reconocidos por } \\
\text { aquellos que tienen la } \\
\text { experiencia con el } \\
\text { fenómeno en estudio. }\end{array}$ \\
\hline
\end{tabular}


REVISTADE SALUD PÚBLICA· Volumen 9 (4), Diciembre 2007

Finfgeld (12) indica que para iniciar el estudio de metasíntesis hay que delimitar el tópico del área de interés del investigador. El conocimiento previo y de estudios anteriores realizados por el grupo investigador es un insumo que permite capturar la totalidad del fenómeno de interés.

Muestreo: Finfgeld (12) indica que no existe un consenso frente a cuales son las mejores fuentes de datos que se requieren para la metasíntesis. Sin embargo muchos investigadores insisten en usar solo los hallazgos de investigaciones publicadas ya que estos han pasado por revisiones de alta calidad. Alternativamente, Beck (22) indica la importancia de utilizar los reportes de investigaciones inéditas u originales para identificar hallazgos mucho más específicos que lo que se menciona en un artículo de investigación. Frente a este planteamiento Finfgeld $(13,14)$ expresa las dificultades a que puede verse avocado el investigador por la ubicación de los reportes de estudios inéditos y los costos que implica llegar a las fuentes primarias.

Por otro lado, Sandelowski (23-25) afirma que existe el riesgo de que los criterios de selección sean muy restrictivos y se excluya información valiosa. Ante esta posibilidad se sugieren dos criterios de selección fundamentales. El primero consiste en que los reportes de las investigaciones muestren que éstas fueron conducidas utilizando métodos cualitativos ampliamente aceptados. El segundo, que los hallazgos de las investigaciones estén soportados por las fuentes primarias $(26,27)$.

Tamaño de la muestra: Revisando estudios producto de Metasíntesis se encuentra que no hay un estándar que determine el tamaño de la muestra (28-30). Esta puede variar según sea el objetivo de la metasíntesis. Schreiber (2) considera que para investigaciones cuyo fin sea indagar sobre conceptos particulares en contextos circunscritos se requiere una muestra más pequeña que para aquellos estudios que buscan explorar de forma amplia fenómenos. Sin embargo, el tamaño de la muestra no está determinado por la cantidad de estudios sino por el contenido de la información que estos arrojan y el tipo de metasíntesis seleccionado.

No sólo un gran número de estudios pueden ser significativos para el logro de los resultados de la investigación. Según Barroso (31), Paterson (21) y Sandelowsky (23), las muestras demasiado grandes pueden impedir el análisis profundo amenazando la validez interpretativa de los hallazgos y resultando en amplias generalizaciones que son de poca utilidad en la práctica. 
Análisis de datos: Morse (32) sugiere que los hallazgos de los estudios deben ser analizados a través de temas, categorías y procesos, más allá que trabajar con los conceptos por sí mismos; esto permite comparar e interpretar los datos de varios estudios.

Finfgeld (12) considera que los hallazgos provenientes de diferentes perspectivas epistemológicas deben ser analizados por separado, mientras que otros autores como Strauss (33) expresan que ésta es una restricción innecesaria y que por el contrario la combinación de hallazgos generados por diferentes metodologías, pueden ser complementarios.

Además del análisis de los datos, la metasíntesis permite al investigador generar una serie de supuestos relacionados con los hallazgos de los estudios y las interpretaciones que se han hecho sobre estos. En particular, ésta facilita la ampliación de la interpretación de lo que se ha estudiado a lo que no ha sido estudiado y la especulación sobre el por qué es así, tal como lo indica Paterson (21).

Resultados: en la mayor parte de los productos de metasíntesis no es posible predecir el grado en el cual se pueda explicar, describir o construir el nuevo conocimiento, para ello se espera que los resultados del análisis sean interpretados de manera individual y colectiva, como ya se mencionó. Al realizar este proceso los investigadores comúnmente observan que existen brechas, defectos y limitaciones en los estudios de tipo cualitativo, lo que probablemente, les llevará a analizar críticamente y concluir que no hay un conocimiento suficiente sobre un fenómeno en particular.

Sin embargo, se considera que los resultados de la metasíntesis permiten analizar aspectos particulares del fenómeno que se desconocían, por lo cual el éxito de esta metodología no radica únicamente en la obtención de una visión más comprehensiva y totalitaria del fenómeno, sino en la producción de información útil para su conocimiento. Tal como lo afirma, Paterson (21) "la llave a un esfuerzo exitoso de metasíntesis radica en el reconocimiento de que las ganancias pequeñas pueden ser tan importantes como las grandes y que pueden ser más útiles, en este largo camino, mejores formas de teorización con respecto a aspectos más pequeños de un campo, que las teorías completamente fundamentadas en otras previas"

\section{REFERENCIAS}

1. Galvez A. Métodos contemporáneos de síntesis científica: una propuesta de homogeneidad, clasificación de las investigaciones por su diseño y utilidad. Index deEnfermería 2003; 12(43):45-49. 
REVISTADE SALUD PÚBLICA· Volumen 9 (4), Diciembre 2007

2. Schreiber R, Crooks D, Stern P. Qualitative meta-analysis. In Morse JM. Completing a qualitative project: Details and dialogue. Thousand Oaks: SAGE; 1997: 311-326.

3. Schreiber, R., Qualitative meta-analysis. In Morse JM. Qualitative Nursing Research: A contemporary dialogue. London: SAGE; 1989: 311-327

4. Noblit G. The prospects of applied ethnography for education: A sociology of knowledge interpretation. Educational Evaluation and Policy Analysis 1984; 6(1): 95-101.

5. Noblit G, Hare R. Meta-ethnography: Synthesizing qualitative studies, the shifting perspectives model of chronic illness. Journal of Nursing Scholarship 2001; 33: 21-26.

6. Estabrooks C, Field PA, Morse JM. Aggregating qualitative findings: An approach to theory development. Qualitative Health Research 1994; 4: 503-511.

7. Gálvez A. Actualidad de la enfermería basada en la evidencia, superando la evidencia científica: la metasíntesis. Index Enfermería digital 2003; 40-41. [Internet]. Disponible en: http://www.index-f.com/index-enfermería/40-41revista/4041_articulo_7-8.php. Consultado: Diciembre del 2006.

8. Gálvez A. Evidencias, pruebas científicas y enfermería. Reflexión en voz baja y pensamientos inconfesables. Enfermería Global. [Internet]. Revista electrónica semestral de enfermería 2003; noviembre (3). Disponible en www.um/es/eglobal. Consultado: Diciembre 2006.

9. Gálvez A. Práctica Clínica Basada en la Evidencia: Una aproximación bibliográfica. Desarrollo Científico de Enfermería 2000; 8(4): 119-121.

10. Gálvez A. Lectura crítica de un estudio cualitativo interpretativo. Index de Enfermería 2003; 12(42):39-43.

11. Gu Jifa T. Meta-synthesis Knowledge System (MSKS). Institute of systems Science, AMSS, CAS. Bijing. 2001; 21-23.

12. Finfgeld DL. Metasynthesis: The state of the art-So far. Qualitative Health Research 2003; 13: 893-904.

13. Finfgeld DL. Courage as a process of pushing beyond the struggle. Qualitative Health Research 1999; 9: 803-814.

14. Finfgeld DL. Self-resolution of drug and alcohol problems: A synthesis on presence, touch and listening. Journal of Addictions Nursing 2000; 12: 65-72.

15. Kearney MH. Ready to wear: Discovering grounded formal theory. Research in Nursing and Health 1998; 21: 179-186.

16. Kearney MH. New directions in grounded formal theory. In Schreiber R, Stern PN. Using grounded theory in nursing. New York: Springer 2001; 227-246.

17. Kearney MH. Levels and applications of qualitative research evidence. Research in Nursing \& Health 2001; 24: 145-153.

18. Kearney MH. Enduring love: A grounded formal theory of women's experience of domestic violence. Research in Nursing \& Health 2001; 24: 270-282. 
19. Schreiber RS, Stern PN. Using grounded theory in nursing. New York: Springer, 2001: 25-29.

20. Egger M, Smith GD. Meta-analysis: Potentials and promise. Bristish Medical Journal 2001;315: 1571-1574.

21. Paterson B. Meta-study of Qualitative Health Research. A practical guide to metaanalysis and meta-synthesis .Thousand Oaks: Sage; 2001: 113.

22. Beck CT. Caring within nursing education: a metasynthesis. Journal of Nursing Education 2001; 40: 101-109.

23. Sandelowski M. Qualitative Metasynthesis issues and techniques. Research in nursing and health 1997; 20: 365-371.

24. Sandelowski M, Barroso J. Writing the proposal for a qualitative research methodology project. Qualitative Health Research 2003; 13: 781-820.

25. Sandelowski, M., Barroso, J. Creating meta-summaries of qualitative findings. Nursing Research 2003; 52: 226-231.

26. Sandelowski M, Barroso J. Toward a meta-synthesis of qualitative findings on motherhood in HIV-positive women. Research in Nursing \& Health 2003; 26 : 153-170.

27. Sandelowski M. On the aesthetics of qualitative research. Journal of Nursing Scholarship 1995; 27: 205-209.

28. Campbell R, Pound P, Pope C, Britten N, Pill R, Morgan M, Donovan J. Evaluating meta-ethnography: A synthesis of qualitative research on lay experiences of diabetes and diabetes care. Social Science \& Medicine 2003; 56: 671-684.

29. Hess E. The collaboration process in professional development schools: Results of a meta-ethnography 1990-1998. Journal of Teacher Education 2002; 53(11): 5568.

30. Joachim G, Acorn S. Living with chronic illness: The interface of stigma and normalization. Canadian Journal of Nursing Research 2000; 32(3): 37-48.

31. Barroso J, Powell-Cope GM. Metasynthesis of qualitative research on living with HIV infection. Qualitaive Health Research 2004; 10: 340-353.

32. Morse J, Johnson J. The illness experience: Dimensions of suffering. Newbury Park: Sage; 1991.

33. Strauss A, Corbin J. Grounded theory methodology: An overview. In Denzin N, Lincoln Y. Handbook of Qualitative Research. Thousand Oaks: Sage; 1994: 273285. 